\section{RMD Open}

Rheumatic \& Musculoskeletal Diseases

\title{
Autoantigen TRIM21/Ro52 is expressed on the surface of antigen-presenting cells and its enhanced expression in Sjögren's syndrome is associated with B cell hyperactivity and type I interferon activity
}

Maarten R Hillen (D, , ${ }^{1,2}$ Katia Urso, ${ }^{3}$ Emma Koppe, ${ }^{3}$ Ana Pinheiro Lopes, ${ }^{1,2}$ Sofie L M Blokland, ${ }^{1,2}$ Aridaman Pandit (D), ${ }^{1,2}$ Tom Slocombe, ${ }^{3}$ André van Maurik, ${ }^{4}$ Joel A G van Roon, ${ }^{1,2}$ Timothy R D J Radstake ${ }^{1,3}$

Tripartite motif-containing protein 21 (TRIM21)/Ro52 is an immunoglobulin receptor that together with Ro60 makes up Sjögren's syndrome-antigen A (SSA). A $60 \%-80 \%$ of patients with primary Sjögren's syndrome (pSS) have anti-SSA autoantibodies and they form part of the pSS diagnosis. TRIM21 is a cytosolic Fc-receptor that mediates neutralisation of opsonised viruses and other particles, acting as a last line of defence against viruses that are recognised by antibodies but still manage to penetrate the cell membrane. ${ }^{1}$ In addition, TRIM21 regulates type I interferon (IFN) responses by mediating either stabilisation or degradation of IFN regulatory factors. ${ }^{1}{ }^{2}$ Uniquely, it has a very broad antibody specificity and recognises IgA, IgG and IgM antibodies with high affinity, allowing TRIM21 to be effective against a large range of viruses. ${ }^{3}$ Alternatively, TRIM21 can be triggered by immune complexes containing non-viral particles such as cellular debris or nucleic acids, ${ }^{4}$ leading to rapid production of proinflammatory mediators including type I IFNs. ${ }^{2}$ Interestingly, mice immunised with TRIM21 develop anti-TRIM21 autoantibodies and salivary gland inflammation, while transfer of serum from anti-TRIM21-positive mice to naïve mice induces salivary gland dysfunction, ${ }^{5}$ suggesting a direct link between anti-TRIM21 autoantibodies and induction of Sjögren's-like symptoms.

Considering the relevance of TRIM21 as autoantigen in pSS and its role in regulating IFN signalling, we here investigated its

\section{Key messages}

What is already known on this subject

- TRIM21/R052 is a major autoantibody target in pSS and regulates downstream signalling of type I IFNs.

- Apoptosis-associated surface expression of TRIM21 on non-immune cells is associated with enhanced binding of anti-Ro52 autoantibodies.

What this study adds

- Enhanced expression of TRIM21 in type I IFNproducing immune cells from patients with pSS is associated with an IFN signature and B cell hyperactivity.

- TRIM21 is expressed on the cell surface of nonapoptotic antigen-presenting cells and its expression is enhanced by IFN- $\beta$ exposure.

How might this impact on clinical practice

- Elucidating the relationship between type I IFNs and anti-SSA autoantibodies in pSS may aid identification of promising therapeutic avenues.

expression in the major type I IFNproducing immune cells, plasmacytoid dendritic cells (pDCs) and monocytes, from patients with pSS. In addition, we investigated TRIM21 expression in salivary gland tissue. For pDCs and salivary gland tissues, RNA sequencing and analysis were performed separately as previously described. $^{6} \quad{ }^{7}$ For monocytes, TRIM21 expression was assessed using quantitative PCR (online supplementary methods) We observed increased mRNA expression of 
TRIM21 in purified pDCs from patients with pSS compared to both healthy controls and patients with non-Sjögren's sicca (nSS), as previously described by our group. ${ }^{6}$ Similarly, pSS patients showed increased expression of TRIM21 in monocytes compared to HC. Additionally, we observed that salivary glands from patients with pSS expressed more TRIM21 compared to patients with nSS (figure 1A). TRIM21 expression was associated with systemic B cell hyperactivity, positivity for autoantibodies against SSA and the expression of type I IFN-induced genes (ie, the IFN score) (online supplementary figure 1). We also observed a significant difference in TRIM21 expression between SSB-positive and SBB-negative pSS patients in pDCs $(\mathrm{p}=0.047)$. No differences in TRIM21 expressed were observed between rheumatoid factor-positive and rheumatoid factor-negative pSS patients in any of the compartments analysed (not shown).

We next set out to confirm this enhanced expression at the protein level in circulating cells in an independent cohort (online supplementary table 1, online supplementary figure 2). However, intracellular (IC) staining by flow cytometry of both pDCs and monocytes against TRIM21 revealed no differences between pSS and healthy controls (HC) donors (figure 1B,C). ImageStream analysis showed that TRIM21 expression is located in the cytosol and in vesicles, consistent with literature $^{8}$ (figure 1D,E). Strikingly, we also observed expression of TRIM21 in nonpermeabilised monocytes, suggesting surface expression. pDCs also showed cell surface expression in several donors. To verify that this apparent TRIM21 surface expression was not an artefact due to leakage of cytosolic TRIM21, we excluded cells with a disrupted cell membrane from the analysis using live/dead staining. When comparing surface expression of TRIM21, pSS patients showed increased expression on monocytes compared to healthy donors (figure 1F,G). To investigate whether TRIM21 surface expression was also present on other immune cells, we assessed CD11c-expressing classical dendritic cells (cDCs) and $\mathrm{T}$ cells within the stained peripheral blood mononuclear cells (PBMCs). cDCs showed a clear surface TRIM21 signal, while expression on $\mathrm{T}$ cells was very low or undetectable (online supple mentary figure 3 ). Consistent with the flow cytometry data, ImageStream analysis of freshly isolated PBMCs resulted in a clear signal after surface staining for TRIM21, which was mainly localised in vesicle-like structures suggesting internalisation (online supple mentary figure 3 ).

As the expression of TRIM21 was associated with the IFN score in the patients, we investigated whether type I IFNs would enhance surface TRIM21 expression. After stimulation of PBMCs with IFN- $\beta$ for 24 hours, we observed a robust increase in TRIM21 mRNA expression (online supplementary fig ure 4). In addition, IFN- $\beta$ stimulation resulted in enhanced surface expression of TRIM21 on monocytes (figure 2A). To further verify that surface expression of TRIM21 is enhanced by IFN- $\beta$, we used extracellular protein biotinylation. After stimulation, total PBMCs were lysed and the extracellular proteins were separated from the IC proteins and evaluated using western blot. Corroborating the previous results, TRIM21 expression was increased upon IFN- $\beta$ stimulation in both the intracellular and extracellular fractions (figure 2B,C).

The presence of anti-SSA antibodies is a hallmark of Sjögren's syndrome and associated with increased B cell hyperactivity. In addition, evidence suggests that antibodies against TRIM21 directly contribute to pSS immunopathology and symptoms. ${ }^{5}$ Interestingly, most anti-TRIM21 antibodies in pSS patients recognise and block domains critical for the antiinflammatory effects of TRIM $21,{ }^{9}$ while its proinflammatory effects that lead to enhanced type I IFN production remain intact. This is corroborated by the strong association between anti-SSA positivity and the presence of an IFN signature in patients with pSS. Although we could not distinguish between positivity for anti-Ro52 and anti-Ro60 in the current study, the observed association between increased TRIM21 expression and positivity for SSA and the IFN score further suggests that TRIM21 overexpression perpetuates inflammation via intracellular pathways that enhance type-I IFN production. This is in good agreement with studies that identify TRIM21 as a central mediator in anti-viral immunity, where it drives production of type I IFN and other pro-inflammatory cytokines via ubiquitination of IFN regulatory factors and mediators in the nuclear factor kappa-light-chain-enhancer of activated B cells (NF-kB) pathway. ${ }^{2} 310$

Enhanced TRIM21 surface expression may also drive inflammation in pSS. Surface expression of TRIM21 was hitherto only observed in non-immune cells that undergo apoptosis, where it was linked to enhanced binding of anti-SSA antibodies. ${ }^{11}$ As we excluded dead and dying cells from our flow cytometry analyses and corroborated our results in freshly isolated PBMCs, it is unlikely that the observed surface expression represents apoptotic cells. Yet, TRIM21 surface expression may well facilitate binding of anti-SSA antibodies and cognate SSA-reactive $\mathrm{B}$ cells, driving $\mathrm{B}$ cell activation and production of anti-SSA autoantibodies, which is in-line with the association between increased TRIM21 expression and anti-SSA positivity. 




Monocytes



Salivary glands



B



D CD303 / Isotype
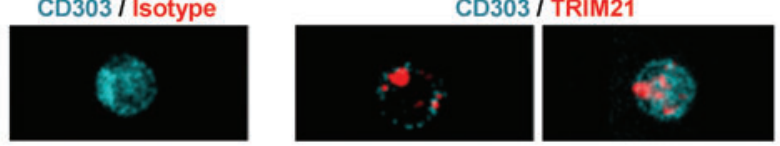

F
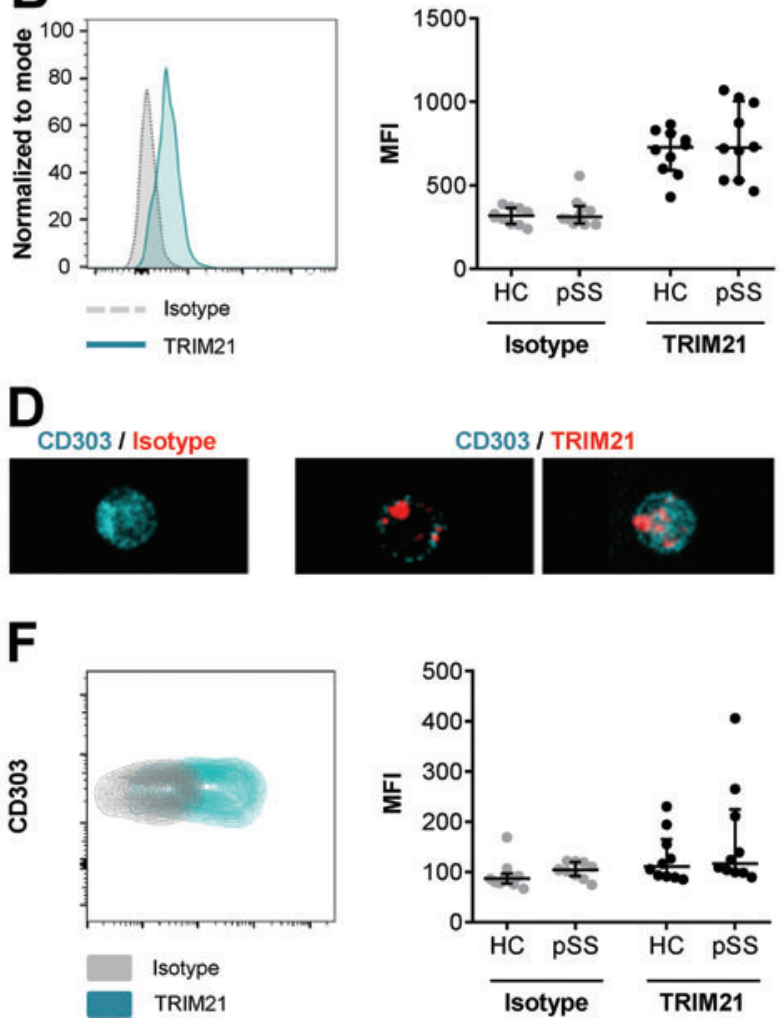

C


E


G
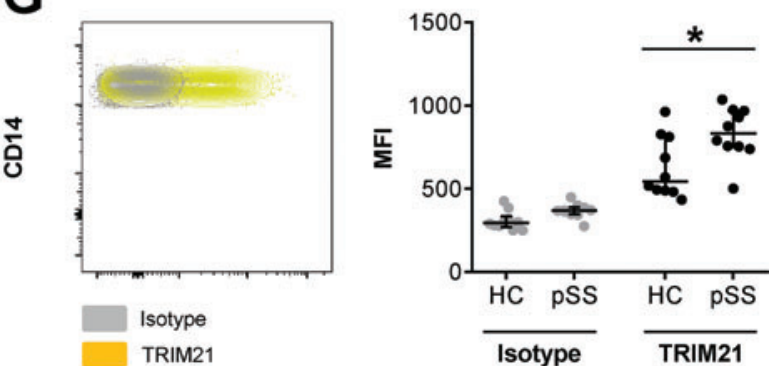

Figure 1 Tripartite motif-containing protein 21 (TRIM21) is overexpressed on the cell surface of monocytes from patients with primary Sjögren's syndrome (pSS). TRIM21 gene expression was quantified in purified circulating pDCs and salivary gland tissue using RNAsequencing and in monocytes using QPCR. RNA sequencing data is depicted as counts per million reads (cpm) or normalised read counts (NRC; 2 log scale). All pSS patients met the 2002 AECG classification criteria, nSS patients presented with dryness complaints without a known cause, did not have any generalised autoimmune disease and did not fulfil the classification criteria for pSS (A). Using flow cytometry on thawed total PBMCs, intracellular (IC) expression of TRIM21 was measured in

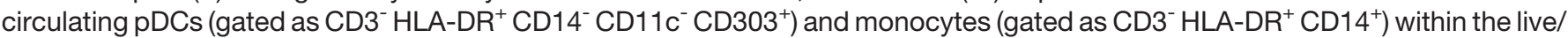
dead staining negative cells (B,C). IC localisation of TRIM21 expression was visualised using ImageStream, representative figures of pDCs (D) and monocytes (E) are shown. TRIM21 surface expression was quantified on pDCs (F) and monocytes (G) within total PBMCs. All data points represent individual donors, data are depicted as median \pm IQR. Flow data are represented using median fluorescent intensity. ${ }^{*}$ and ${ }^{* * *}$ indicate $p<0.05$ and $p<0.001$, respectively.

The function of surface TRIM21 remains to be determined. We hypothesise that on the cell surface, TRIM21 acts as an Fc-receptor that activates its ubiquitin-ligase subunit when ligated with immune complexes and subsequently modifies other mediators localised in its proximity. Given its high affinity, surface TRIM21 would be quickly internalised in recycling endosomes together with its potential targets of ubiquitination, allowing it to drive cellular activation and production of IFNs, similarly to its described function in the cytosol. This is in-line with the localisation of TRIM21 in vesicles after surface staining of 
A
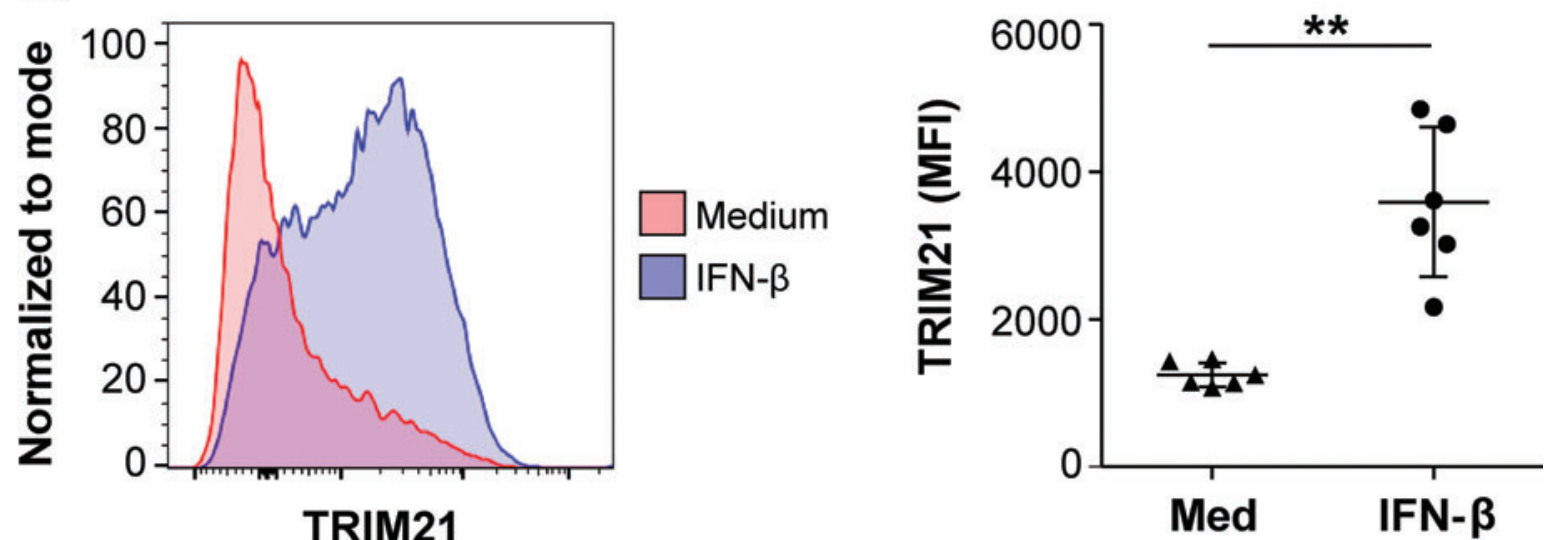

B

\section{TRIM21}
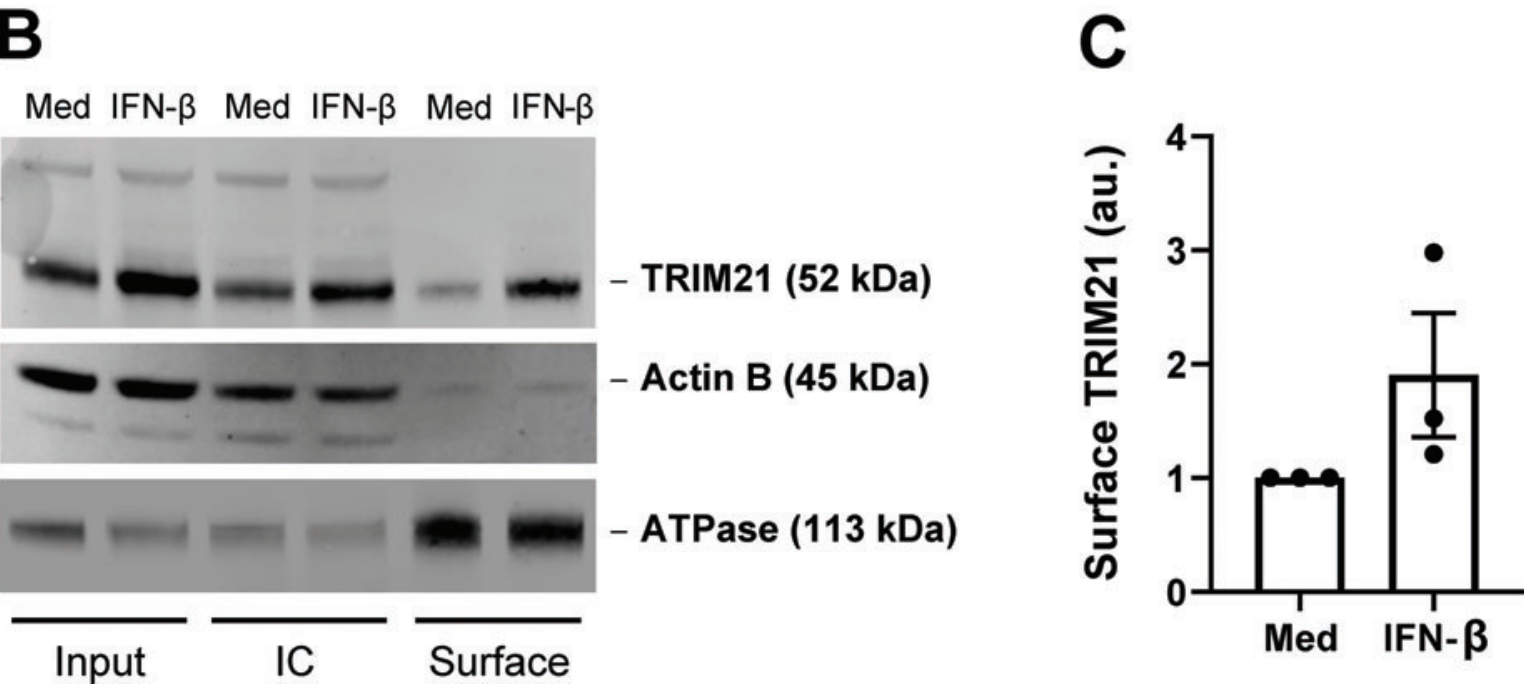

Figure 2 Surface expression of tripartite motif-containing protein 21 (TRIM21) on monocytes is enhanced by interferon- $\beta$ (IFN- $\beta$ ). Freshly isolated HC PBMCs were cultured for 24 hours in the absence or presence of $50 \mathrm{ng} / \mathrm{mL}$ of IFN- $\beta$. Surface TRIM21 expression was analysed on monocytes (gated as CD3 ${ }^{-} \mathrm{HLA}-\mathrm{DR}^{+} \mathrm{CD} 14^{+} \mathrm{CD} 16^{-}$) using flow cytometry (A). In total PBMCs, surface proteins were separated from intracellular (IC) proteins and TRIM21 contents were analysed using western blot. Detection of actin- $\beta$ confirmed minimal contamination of IC proteins in the surface protein fraction. Actin- $\beta$ and sodium/potassium ATPase were used as loading controls for IC and surface protein fraction, respectively. (B) Results representative for three independent experiments are shown. Extracellular TRIM21 expression upon stimulation was quantified relative to the unstimulated condition and depicted in arbitrary units (au.) (C). Flow data are represented using median fluorescent intensity (MFI). All dots represent independent donors, data are depicted as mean \pm SEM, ${ }^{*}$ and ${ }^{* *}$ indicate $p<0.05$ and $p<0.01$, respectively.

PBMCs and with the observed decrease in surface TRIM21 expression after exposure of monocytes to immune complexes (unpublished data). Further research should elucidate the function of surface TRIM21 expression and its relevance for anti-SSA autoantibody production in pSS and other systemic autoimmune diseases.

\footnotetext{
Author affiliations

${ }^{1}$ Centre for Translational Immunology, University Medical Center Utrecht, Utrecht University, Utrecht, the Netherlands

${ }^{2}$ Department of Rheumatology \& Clinical Immunology, University Medical Center Utrecht, Utrecht University, Utrecht, the Netherlands

${ }^{3}$ GSK Immunology Network and Adaptive Immunity Research Unit, GlaxoSmithKline, Stevenage, UK

${ }^{4}$ Clinical Pharmacology and Experimental Medicine, GlaxoSmithKline, Stevenage, UK
}

Acknowledgements We would like to thank Dr Sarita Hartgring, Dr Fréderique van Vliet-Moret, Dr Cornelis Bekker, Catharina Wichers and Kim van der Wurff-Jacobs for help with sample isolation and help with data analysis. We are grateful to Dr Aike Kruize for his help with patient inclusion and gathering of clinical data.

Contributors MRH, KU, EK, AvM, JAGvR and TRDJR made substantial contributions to conception and design. $\mathrm{MH}, \mathrm{KU}, \mathrm{SB}$, and TS were involved in sample collection. $\mathrm{MRH}, \mathrm{KU}$, and AP performed data analysis. MRH and KU drafted the paper. All authors were involved in data interpretation and revision of the paper for important intellectual content. All authors approved the final manuscript.

Funding This work was in part funded by GlaxoSmithKline. MH was supported by the Dutch Arthritis Society (ReumaNederland, grant nr 17-2-301).

Competing interests TR is a principal investigator in the Immunology Catalyst programme at GlaxoSmithKline, which is an independent research programme. He did not receive any financial support other than the research funding for the current project. The other authors have declared no conflicts of interest.

Patient consent for publication Not required. 
Provenance and peer review Not commissioned; externally peer reviewed.

Open access This is an open access article distributed in accordance with the Creative Commons Attribution Non Commercial (CC BY-NC 4.0) license, which permits others to distribute, remix, adapt, build upon this work non-commercially, and license their derivative works on different terms, provided the original work is properly cited, appropriate credit is given, any changes made indicated, and the use is non-commercial. See: http://creativecommons.org/licenses/by-nc/4.0/.

ORCID iDs

Maarten R Hillen http://orcid.org/0000-0002-9505-3468

Aridaman Pandit http://orcid.org/0000-0003-2057-9737

\section{REFERENCES}

1 Espinosa A, Dardalhon V, Brauner S, et al. Loss of the lupus autoantigen Ro52/TRIM21 induces tissue inflammation and systemic autoimmunity by disregulating the IL-23-Th17 pathway. J Exp Med 2009;206:1661-71.

2 McEwan WA, Tam JCH, Watkinson RE, et al. Intracellular antibody-bound pathogens stimulate immune signaling via the FC receptor TRIM21. Nat Immunol 2013;14:327-36.

3 Bidgood SR, Tam JCH, McEwan WA, et al. Translocalized IgA mediates neutralization and stimulates innate immunity inside infected cells. PNAS 2014;111:13463-8.

4 Monteith AJ, Kang S, Scott E, et al. Defects in lysosomal maturation facilitate the activation of innate sensors in systemic lupus erythematosus. PNAS 2016;113:E2142-51.
5 Szczerba BM, Kaplonek P, Wolska N, et al. Interaction between innate immunity and Ro52-induced antibody causes Sjögren's syndrome-like disorder in mice. Ann Rheum Dis 2016;75:617-22.

6 Hillen MR, Pandit A, Blokland SLM, et al. Plasmacytoid DCs from patients with Sjögren's syndrome are transcriptionally primed for enhanced pro-inflammatory cytokine production. Front Immunol 2019;10:2096

7 Blokland SLM, van Vliet-moret FM, Hillen MR, et al. Epigenetically quantified immune cells in salivary glands of Sjögren's syndrome patients: a novel tool that detects robust correlations of $\mathrm{T}$ follicular helper cells with immunopathology. Rheumatology (Oxford) 2019;366:321.

8 Tanaka M, Tanji K, Niida M, et al. Dynamic movements of Ro52 cytoplasmic bodies along microtubules. Histochem Cell Biol 2010;133:273-84.

9 Espinosa A, Hennig J, Ambrosi A, et al. Anti-Ro52 autoantibodies from patients with Sjögren's syndrome inhibit the Ro52 E3 ligase activity by blocking the E3/E2 interface. J Biol Chem 2011;286:36478-91.

10 Watkinson RE, McEwan WA, Tam JC, et al. TRIM21 promotes cGAS and RIG-I sensing of viral genomes during infection by antibody-opsonized virus. PLoS Pathog 2015;11: e1005253.

11 Ohlsson M, Jonsson R, Brokstad KA. Subcellular redistribution and surface exposure of the Ro52, Ro60 and La48 autoantigens during poptosis in human ductal epithelial cells: a possible mechanism in the pathogenesis of Sjögren's syndrome. Scand J Immunol 2002;56:456-69. 